\title{
Miniature optical fiber sensors using surface enhanced Raman spectroscopy (SERS) for remote biochemical sensing
}

\author{
Aditya H. Pandya ${ }^{1}$, Joseph C. Kumaradas ${ }^{1}$, and Alexandre Douplik ${ }^{1,2,3^{*}}$ \\ ${ }^{1}$ Department of Physics, Ryerson University, Toronto, ON, M5B 2K3, Canada \\ 2 Institute for Biomedical Engineering, Science and Technology (iBEST), St. Michael's hospital, Toronto, ON, M5B 1W8, \\ Canada \\ ${ }^{3}$ Keenan Research Centre for Biomedical Science of St. Michael's Hospital, Toronto, ON, M5B 1T8, Canada \\ * e-mail: douplik@ryerson.ca
}

\begin{abstract}
In this study, we present facile fabrication of a miniaturized remote sensing SERS platform using highly tunable Nano-Sphere Lithography (NSL) technique. Using $200 \mu \mathrm{m}$ diameter optical fibers with high numerical aperture (0.5NA), the SERS enhancement of remote sensing was found to be $98 \%$ of direct sensing configuration. Standard silica optical fibers were used for remote sensing using SERS without additional need of optical filtering to mitigate fluorescence and Raman background of these fibers which allows fabrication of miniaturized remote sensing platforms that can be used for remote biochemical sensing. (C) 2019 Journal of Biomedical Photonics \& Engineering.
\end{abstract}

Keywords: SERS; Plasmonics; Nanoparticles; Nanosphere lithography; Remote fiber sensing.

Paper \#3303 received 13 Nov 2018; revised manuscript received 26 Jan 2019; accepted for publication 27 Jan 2019; published online 9 Feb 2019. doi: 10.18287/JBPE19.01.010301.

\section{Introduction}

Raman spectroscopy (RS) is a widely used analytical technique that provides a molecular vibrational fingerprint of the analyte being examined based on inelastic scattering of light [1]. It has been successfully used in a wide range of applications such as detecting explosives, process analytical techniques and, medical endoscopy [2-5]. Remote sensing using RS poses a major challenge as it is a very weak process and intrinsic fluorescence routinely interferes with RS data collection because it is 4 to 5 orders of magnitude larger in magnitude [6]. Furthermore, miniaturizing RS probes in a form factor less than a millimeter leads to low signal throughput limiting the detection sensitivity. Remote sensing in microfluidic platforms, endoscopic devices, and industrial process analytical technologies is routinely desired. Hence, miniaturization of Raman probes is necessary while maintaining proper signal-tobackground ratio allowing for efficient collection of RS spectra.

Standard silica based optical fibers produce large fluorescence background [7]. This is eliminated by using optical filters to limit excited optical fiber fluorescence through a laser line filter and backscattered Rayleigh light using a dichroic/notch filter at the fiber tip [7] These filters are expensive to manufacture and hard to miniaturize. Sapphire based optical fibers (SOF) and photonic crystal/hollow core optical fibers (PCF) have been proposed to minimize the background $[8,9]$. Both SF and PCF are expensive to handle and manufacture while PCFs also have lower collection efficiencies and limited operational bandwidth. Thus, standard silica based optical fibers are lucrative for creating low cost (a few dollars/meter) and disposable RS platforms due to their wide availability with varying collection efficiencies.

Surface Enhanced Raman Spectroscopy (SERS) has been used to enhance the inherently weak Raman scattering and the large enhancement factors by SERS have resulted in lower limits of detection (up to single molecule sensitivity) [10-12]. SERS has been performed primarily using nanoparticles suspended in a solution mixed with an analyte or nanoparticles localized on a substrate that is incubated with the analyte $[12,13]$. SERS substrates need to be designed for single use to 
limit cross contamination and to avoid physical and chemical degradation of substrates over time [14]. Using SERS with standard silica optical fibers presents a unique opportunity to extend the usage of optical fibers toward unfiltered RS probes.

Nanosphere lithography (NSL) is a versatile technique for fabricating nanostructures [15]. At first, a tightly packed array of nanospheres is created using dip coating, spin coating or assembly at the interface of two media [16]. After the self-assembly process, SERS active substrates are fabricated using two approaches: 1) Through deposition of a metal film over the nanosphere array to create metal film over nanospheres (MFON) substrates, and 2) Depositing a metal film over the nanosphere array and then removing nanosphere array mask to create periodic nanotriangle arrays matching the shape of the gap in between the tightly packed nanosphere array. Periodic nanotriangle arrays provide wavelength tunability and control of surface area that can be more suitable for quantitative calculation of enhancement factors and performing theoretical calculations of electromagnetic enhancements [17]. Since the size of nanospheres used as mask can be altered, nanoparticle size and distance between particles can be changed which allows for coupling effects between periodic particle arrays allowing for larger enhancements [18]. Thus, NSL is an inexpensive fabrication technique with flexible tunability that can be used for low cost disposable platforms with arbitrary and miniscule geometries.

Recently, more efforts have been focused on using SERS for remote sensing. Various studies have focused on creating optical fibers for remote SERS sensing. These include etched imaging fiber bundles coated with silver films [19], oblique angle deposition of silver films onto fiber tips [20], laser induced self-assembly of Ag nanocubes in a meniscus [21] and, D-shaped fiber tips ablated with femtosecond lasers to create SERS sensors [22]. Trapping nanospheres on fiber surfaces was achieved by Stokes et al. [23] and NSL for SERS on fiber tips was used more recently by Pisco et al. [24]. They focused on improved etching techniques to perform controlled nanoparticle formation and were performed directly on the NSL fabricated SERS tips inside a ferrule and remote sensing was not focused during these studies. A recent study performed by Quero et al. [25] characterized planar substrates using metal film over nanospheres and evaluated background generated by various optical fibers to indicate that fiberoptic optrodes can be created using their approach. Metal film over nanosphere substrates are robust but, they do not allow tunability and the presence of nanospheres on the fiber tips can result in generation of additional background peaks from Polystyrene to interfere with data collection [17, 26]. Moreover, using confocal Raman microscopes, as used by Quero et al., collection of spectra from a large area (such as $200 \mu \mathrm{m}$ optical fibers) is difficult due to rejection of photons because the confocal aperture is smaller than the projected image of the optical fiber on confocal imaging plane.

In this study, optical fiber SERS sensors are fabricated using NSL by removing nanospheres to create nanotriangles. Different Numerical Apertures (NA) were used in a remote sensing configuration using crystal violet $(\mathrm{CV})$ dye as an analyte to test the feasibility of creating a disposal fiber SERS platform. A custom data collection setup was created to acquire spectra from the full diameter of the optical fibers (200 $\mu \mathrm{m})$ to ascertain the projected image of the optical fiber matches the aperture of the collection optical fiber.

\section{Methods}

A custom designed Raman collection setup optimized for fiber excitation and collection was used. A $40 \times$ microscope objective ( 0.65 NA, Motic, China) was used to focus the collimated laser (785 nm, Ondax Inc, USA) into a $30 \mu \mathrm{m}$ spot size on the fiber (Fig. 1D). Since the optical fiber was $200 \mu \mathrm{m}$ in diameter, the excitation spot was centered onto the fiber and a dichroic beamsplitter was used to direct light to and from the sample into a spectrometer. A fiber coupled high throughput spectrometer (16 bit digitization, Hyperflux PRO, Tornado Spectral Systems, Canada) was used to collect the Raman spectra (integration time $1 \mathrm{~s}$ ). The collection was done using a $1 \mathrm{~mm}$ optical fiber due to the magnification achieved using a combination of $40 \times$ microscope objective and a coupling lens which resulted in collection of spectra from the whole diameter of the optical fiber. Calibration of the spectrum was performed using a silicon disc to account for laser variations. Laser power was measured at the sample using a thermal power sensor (PM160T, Thorlabs, USA). The fiber was micro-positioned to achieve the maximum detected signal at the spectrometer.

Standard silica based optical fibers were purchased from Thorlabs (NJ, USA) and were cut to $15 \mathrm{~cm}$ length. Optical fibers were made of silica glass as a concentric core, cladding and coating/buffer (for support) tube. For $200 \mu \mathrm{m}$ core optical fibers used in this study, the cladding is $25 \mu \mathrm{m}$ thick and the coating ranges from $100 \mu \mathrm{m}$ to $275 \mu \mathrm{m}$. Fibers with a range of numerical apertures $(0.22,0.39$ and 0.5$)$ were used. The optical fibers used in this study contain a low $\mathrm{OH}$ concentration which was found to provide the lowest fluorescence/Raman background [27]. A fiber stripper tool was used to strip the coating from both ends. Fiber tips were prepared by scoring them using a fiber scribe (S90R, Thorlabs, NJ, USA) and pulling them to create flat cleaves (Fig. 1A \& B). The prepared tips were sonicated in ethanol solution for 5 minutes and then rinsed off with Miili-Q ultrapure water. The optical fibers were then treated with oxygen plasma ( 5 minutes - High setting, PDC-32 G - 150W, Harrick Plasma, USA) to further prepare the fiber tips for NSL by improving their hydrophilic properties. The prepared tips were immediately soaked in an ultrapure water solution. 

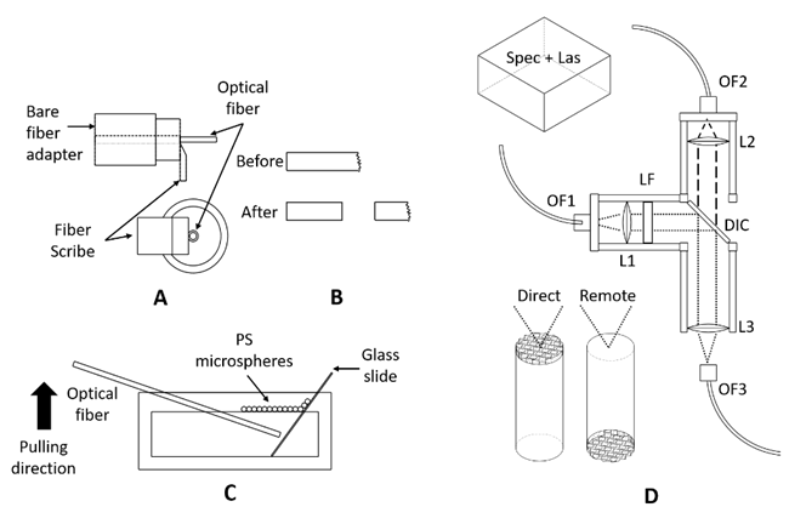

Fig. 1 Fabrication of SERS fibers and RS collection setup (A) Optical fiber guided into a bare fiber adapter with $1 \mathrm{~cm}$ tip protruding through the bore. A fiber scribe scores the fiber tip perpendicularly at the base guided by the ferrule surface. (B) The fiber is then pulled so that it breaks at score line to obtain a flat fiber tip. (C) The plasma treated fiber tip is immersed into a water bath containing a glass slide. PS microspheres are dispersed onto the surface of water through controlled deposition via the glass slide. Upon formation of a monolayer, the optical fiber is pulled out of the water bath to transfer the monolayer onto the fiber tip. (D) Using a $105 \mu \mathrm{m}$ optical fiber (OF1), laser light is collimated using lens (L1) and filtered using a band pass laser line filter (LF). The collimated laser light passes through a dichroic mirror (DIC) which reflects the laser lights on the SERS fiber (OF3) in direct or remote configurations. Red shifted wavelengths passed through the DIC and another lens (L2) focused light into a $1 \mathrm{~mm}$ optical fiber which is connected to a Raman spectrometer.

To perform NSL, a plasma treated microscopic glass slide was placed in a custom Polytetrafluoroethylene (PTFE) tank filled with water and the optical fiber was lowered into the water at an oblique angle 10 using a 3D translation stage (MTS50-Z8, Thorlabs, NJ, USA) as depicted in Fig. 1C. $5 \mu \mathrm{L}$ solution of $50 \%$ ethanol and $50 \%$ polystyrene microsphere solution $(5 \mu \mathrm{m}-4 \mathrm{wt} \%)$ ThermoFisher, USA) was pipetted onto the glass slide and the spheres transferred from the glass slide onto the water surface (Fig. 1C). The process was repeated until the surface was filled with microspheres and an iridescent pattern was obtained. A PTFE bar was used to sweep the surface for a tighter packed monolayer. Once a monolayer was achieved, the optical fiber was pulled out at a steady rate of $10 \mu \mathrm{m} / \mathrm{s}$ as depicted in Fig. 1C. The slow draw of the optical fiber allows the spheres to be coated onto the fiber tip gradually. Sputter coating (Denton Desk IV) was used to coat $25 \mathrm{~nm} \mathrm{Au} \mathrm{layer} \mathrm{on}$ the tightly packed monolayer and ultrasonication was used to remove polystyrene nanospheres.

Fig. 2 shows the background signals from prepared optical fibers. The background from $0.22 \mathrm{NA}$ fiber was consistently distinct from the other fibers showing some interference from fiber coating which was made of acrylate. In general, the region higher than $1300 \mathrm{~cm}^{-1}$ was free from the background generated by the optical fiber. The fluorescence intensity was highest for $0.5 \mathrm{NA}$ below $700 \mathrm{~cm}^{-1}$. The trend was opposite for higher wavenumbers which can be attributed to the variation in material properties of the optical fibers. The laser power at the focal spot was $16 \mathrm{~mW}$ and the power output through the fiber was $3.6 \mathrm{~mW}, 8.2 \mathrm{~mW}$ and $12.4 \mathrm{~mW}$ for $0.2 \mathrm{NA}, 0.39 \mathrm{NA}$ and $0.5 \mathrm{NA}$ respectively.

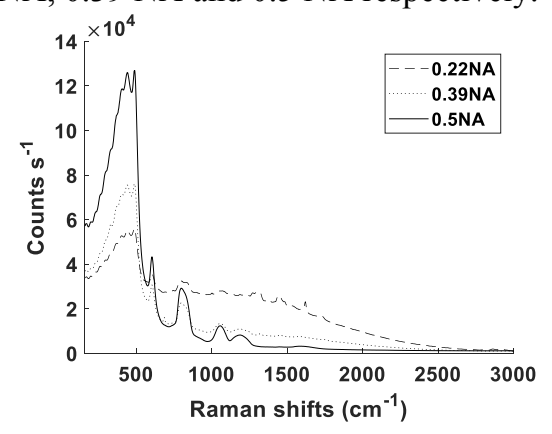

Fig. 2 Background spectra from optical fibers normalized by the integration time.

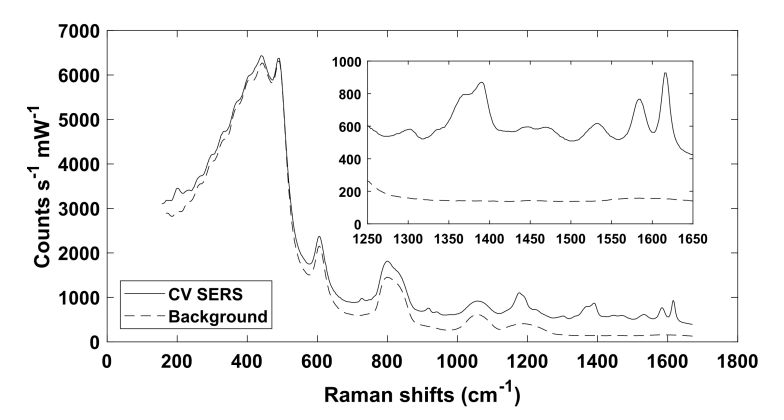

Fig. 3 Background suppression performed via iteratively fitting and removing the known background spectrum from the collected SERS spectrum.

An iterative algorithm with non-negative constraint was used to match the background spectra to the collected SERS spectra. For this study, a fiber background was experimentally obtained, and a simple subtraction resulted in either negative values of intensity or improper suppression of the background. To improve the background suppression, a simple iterative algorithm with non-negative constraints was used to match the background spectra to the collected SERS spectra. The background spectrum was multiplied iteratively by a factor. The factor was initialized to 1 and in each iteration, the factor is increased or decreased by 0.001 depending on the background being larger or smaller than the SERS spectra until the difference is minimized and non-negative (Fig. 3). This allowed to perform an optimized matching of the fiber backgrounds to the SERS spectra. The spectrum was multiplied iteratively by a factor of 0.001 . In each iteration, the background spectrum was subtracted from or added to the SERS spectrum (depending on the background being larger or smaller than the SERS spectra) until the difference was minimized and nonnegative. All the SERS intensity comparisons were performed on raw spectra and background suppression was mainly used for visualizing the peaks. 


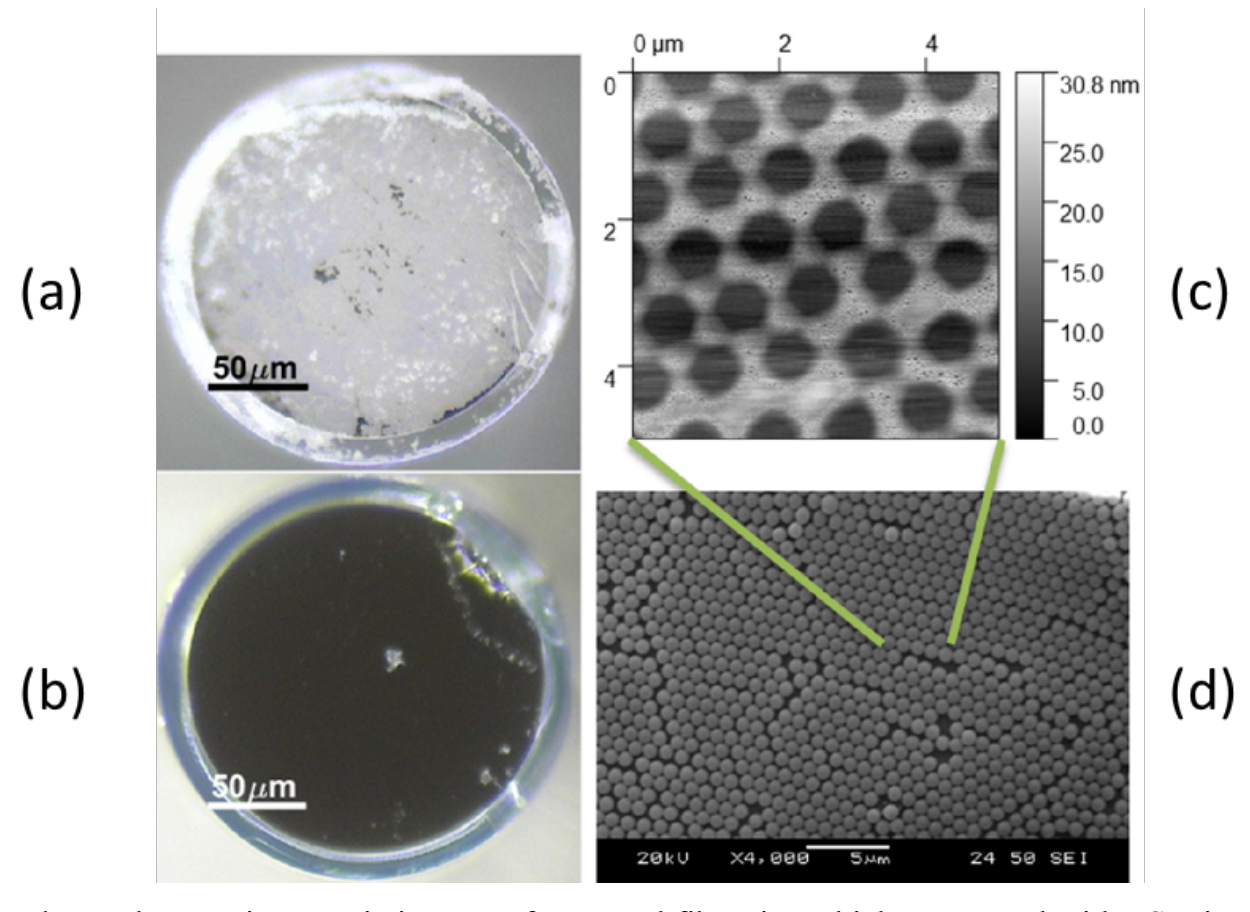

Fig. 4 The left column shows microscopic images of prepared fiber tips which are coated with PS microspheres (a) and uncoated (b). The notch on the top right indicates the location of the fiber score. The right column depicts an AFM image (c) corresponding to the SEM image (d).

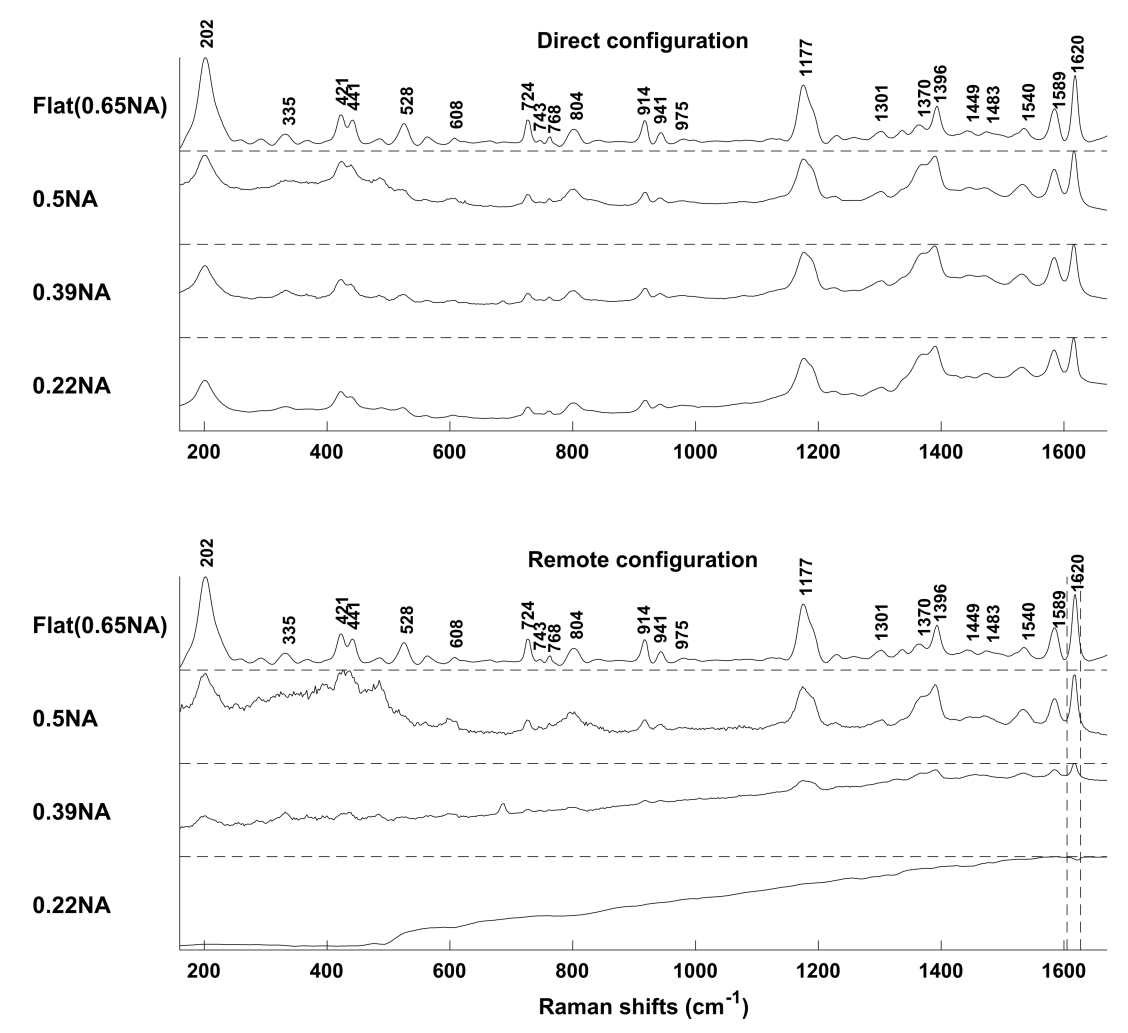

Fig. 5 The spectra are arranged in decreasing order of NA from top to bottom. The topmost spectrum was acquired using a flat substrate fabricated on a microscopic cover slip. The vertical dashed lines in the remote configuration mark the peak that was used to compare the peak intensity for different NA. The spectra are normalized (the scales of each subplot separated by dashed horizontal lines is between 0 and 1) for better depiction of peaks. (a) shows results for direct SERS acquisition configuration and (b) shows results for remote SERS acquisition configuration. 


\section{Results and discussion}

Fig. 4 shows the stages of NSL fabrication and evaluation. The optical fibers were treated with oxygen plasma to prepare the fiber tips for NSL by improving their hydrophilic properties [28] before the fabrication process. This aided in the formation of a monolayer of the polystyrene (PS) microspheres to form an effective mask. Flat cleaved optical fibers were imaged before (Fig. 4b) and after (Fig. 4a) NSL fabrication. The optical image showed full coverage of the fiber tip and corresponding SEM image (Fig. 4d) revealed the monolayer formation. AFM image (Fig. 4c) depicts the hexagonal periodic array of curved triangular $\mathrm{Au}$ nanoparticles created after removal of the polystyrene mask. Line defects (line-like discontinuities in the tightly packed hexagonal lattice structure) were observed in the SEM images which are characteristic to the NSL fabrication process. NSL was also performed on square microscopic cover slips $22 \mathrm{~mm} \times 22 \mathrm{~mm} \times 0.17$ mm made of silica glass.

Following the fabrication of SERS fibers, their performance was evaluated by using Crystal Violet (CV) as an analyte. For maximal efficiency of SERS, the SERS optical fibers and flat substrates were immersed in $244 \mu \mathrm{M}$ Crystal Violet $(\mathrm{CV})$ solution for 3 hours and were rinsed with ultra-pure water for $30 \mathrm{~s}$ to remove non-adsorbed $\mathrm{CV}$ molecules from the surface before performing spectral measurements. For SERS fibers, Raman spectra were collected in the direct (laser incident on the nanofabricated face directly) and remote configurations (laser incident on the remote face opposite to the nanofabricated face). Fig. 5 depicts the spectra obtained through direct and remote configurations. In the direct configurations, acquired spectra for all fiber NAs were within $15 \%$ intensity variation (from the maximum value) confirming the similar performance of the nanostructured tips for all three cases when illuminated directly on the fabricated tips. All the spectra obtained were normalized by the integration time and the laser power coupled through the optical fibers for different NA values. All spectra were corrected for background for visualization purposes as described in the methods section. The optical fibers exhibit strong background below $1100 \mathrm{~cm}^{-1}$. This decreases the quality of Raman spectra below this range. Fig. 6 compares integrated intensity calculated using the tangent skim approach [29] of the peak centered at $1620 \mathrm{~cm}^{-1}$ in the region depicted by the dashed vertical lines in Fig. 5. This was chosen to ensure minimal interference from the background signals generated by the optical fiber. As the collection efficiency of a fiber scales to the square of the NA, a trendline $\left(\mathrm{NA}^{2}\right)$ is presented that depicts the trend of integrated Raman intensity. The integrated peak intensity variation shows good agreement with the trend-line.

For non-SERS spectral collection, fiber probes were immersed in CV solution and spectra were collected from the fibers remotely through the distal end $I_{\mathrm{RS}}^{\text {fiber }}$. These spectra were used as non-SERS spectra for both remote and direct configurations since the fibers did not have any patterned surfaces. For flat glass substrates, instead of immersion, a $10 \mu \mathrm{L}$ of $\mathrm{CV}$ droplet was placed on the substrate for spectral collection $I_{\mathrm{RS}}^{\text {flat }}$. This allowed for data to be acquired for spontaneous Raman scattering with glass background. For SERS spectral collection, spectra were acquired from fabricated flat SERS substrates $I_{\text {SERS }}^{\text {flat }}$ and SERS fiber probes in the remote $\left(I_{\text {SERS }}^{\text {remote }}\right)$ and direct $\left(I_{\text {SERS }}^{\text {direct }}\right)$ configurations. All SERS substrates were immersed in CV solution for 3 hours and then rinsed before collecting spectra.

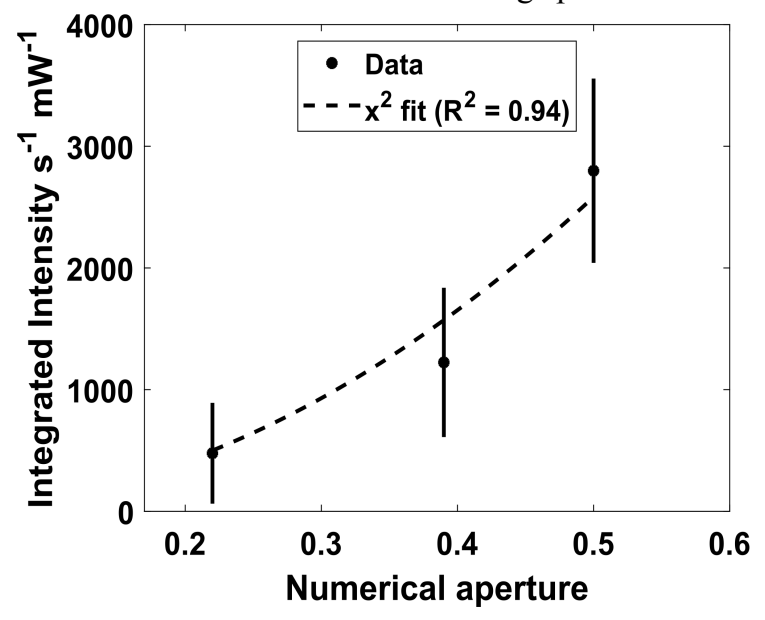

Fig. 6 Integrated peak corresponding to the dashed lines in Fig. 5 depicted against $\mathrm{NA}^{2}$ trendline. The vertical lines for each point represent \pm 1 standard deviation.

For practical performance evaluation, Intensity Enhancement Factors (IEF) were calculated using the following equations

$$
\begin{aligned}
& I E F^{\text {direct }}=\frac{\mathrm{I}_{\mathrm{SERS}}^{\text {direct }}}{\mathrm{I}_{\mathrm{RS}}^{\text {fiber }}}, \\
& I E F^{\text {remote }}=\frac{\mathrm{I}_{\mathrm{SERS}}^{\text {remote }}}{\mathrm{I}_{\mathrm{RS}}^{\text {fiber }}}, \\
& I E F^{\text {flat }}=\frac{\mathrm{I}_{\mathrm{SERS}}^{\text {flat }}}{\mathrm{I}_{\mathrm{RS}}^{\text {flat }}} .
\end{aligned}
$$

$I E F^{\text {direct }}$ was calculated as 31 and 37 for 0.39 NA and $0.5 \mathrm{NA}$ optical fibers respectively. $I E F^{\text {remote }}$ was found to be 11 and 36 for $0.39 \mathrm{NA}$ and $0.5 \mathrm{NA}$ optical fibers respectively. The $I E F^{\text {remote }}$ for $0.5 \mathrm{NA}$ was $98 \%$ of $I E F^{\text {direct }}$ showing that $0.5 \mathrm{NA}$ performed similar in remote and direct configurations. The $I E F^{\text {remote }}$ for 0.39 NA was $35 \%$ of $I E F^{\text {direct }}$ showing that for 0.39 NA, performance degraded in the remote configuration when compared to $0.5 \mathrm{NA}$. The $I E F^{\text {flat }}$ was found to be 30 indicating that the 0.5 NA fiber performed slightly better which can be attributed to the larger area of collection. SERS was shown to partially mitigate the fluorescence background allowing fabrication of unfiltered miniscule Raman probes. Increasing the NA improved the signal-to-background ratio for optimized collection. Since the magnitude of fluorescence scales with the path length of light in the fiber, the fiber 
lengths used in this study were $15 \mathrm{~cm}( \pm 1 \mathrm{~cm})$. These lengths are generally acceptable when being inserted into cavities as well as small microfluidic devices. Flat glass SERS substrates with NSL as well as fiber substrates were fabricated using the same masks. Nonpatterned substrates were also used as control to identify the enhancements achieved. For highest SERS efficiency, a disposable SERS fiber is necessary and thus a low-cost, tunable and reproducible manufacturing process was warranted. NSL technique allows for lowcost, tunable and rapid fabrication procedure with a wide range of enhanced fabrication techniques. Thus, using NSL to create SERS optical fiber sensors is very lucrative to design tailored disposable probes for usage in miniscule and arbitrary sampling environments.

\section{Conclusion}

Disposable SERS fiber probes were fabricated using the NSL technique. Increasing the NA was found to resolve Raman spectra for a wide range of wavenumbers. Direct measurements show similar SERS performance of the optical fibers for all different NAs while remote measurements show that larger NA is required to obtain better Raman characterization. Hence, NA plays a significant role in fabricating SERS fiber probes. A simple iterative matching algorithm with non-negative constraints was sufficient at removing the fluorescence background from the optical fibers. Characterization of SERS optical fibers showed that $0.5 \mathrm{NA}$ had same performance in direct/remote configurations and had the largest enhancement factors indicating advantages of using a high NA optical fiber for SERS measurements. $\mathrm{CV}$ dye was used for a proof of concept to observe the performance of SERS fiber probes in a remote sensing configuration and further experiments should be performed to optimize NSL process and evaluate the limit of detection. This platform can be optimized by changing the size of the microspheres for NSL as well as using advanced etching techniques. Using NSL, bare optical fibers with SERS tips can be easily fabricated and employed for remote sensing. It is envisioned that these fibers can be readily used in miniature environments such as microfluidic devices, and endoscopic devices to perform SERS sensing.

\section{Disclosures}

All authors declare that there is no conflict of interests in this paper.

\section{Acknowledgements}

Authors would like to acknowledge the support for this study from NSERC Discovery grant (Douplik), Ryerson Health Fund, NSERC Engage support, and infrastructural support from Institute for Biomedical Engineering, Science and Technology (IBEST), a partnership between Ryerson University and St. Michael's Hospital (Toronto).

\section{References}

1. C. V Raman, K. S. Krishnan, “A New Type of Secondary Radiation,” Nature 121(3048), 501-502 (1928).

2. X. Fan, I. M. White, "Optofluidic Microsystems for Chemical and Biological Analysis," Nature Photonics 5(10), 591-597 (2011).

3. C. Eliasson, N. A. Macleod, and P. Matousek, "Noninvasive detection of concealed liquid explosives using Raman spectroscopy," Analytical Chemistry 79(21), 8185-8189 (2007).

4. S. Ben-Jaber, W. J. Peveler, R. Quesada-Cabrera, C. W. O. Sol, I. Papakonstantinou, and I. P. Parkin, "Sensitive and specific detection of explosives in solution and vapour by surface-enhanced Raman spectroscopy on silver nanocubes," Nanoscale 9(42), 16459-16466 (2017).

5. K. A. Esmonde-White, M. Cuellar, C. Uerpmann, B. Lenain, and I. R. Lewis, "Raman spectroscopy as a process analytical technology for pharmaceutical manufacturing and bioprocessing," Analytical Bioanalytical Chemistry 409(3), 637-649 (2017).

6. M. Jermyn, J. Desroches, K. Aubertin, K. St-Arnaud, W.-J. Madore, E. De Montigny, M.-C. Guiot, D. Trudel, B. C. Wilson, K. Petrecca, and F. Leblond, "A review of Raman spectroscopy advances with an emphasis on clinical translation challenges in oncology," Physics in Medicine and Biology 61(23), R370-R400 (2016).

7. I. Latka, S. Dochow, C. Krafft, B. Dietzek, and J. Popp, "Fiber optic probes for linear and nonlinear Raman applications - Current trends and future development," Laser \& Photonics Reviews 7(5), 698-731 (2013).

8. L. Yuan, X. Lan, J. Huang, H. Wang, L. Jiang, and H. Xiao, "Comparison of silica and sapphire fiber SERS probes fabricated by a femtosecond laser," IEEE Photonics Technology Letters 26(13), 1299-1302 (2014).

9. S. O. Konorov, C. J. Addison, H. G. Schulze, R. F. B. Turner, and M. W. Blades, "Hollow-core photonic crystal fiber-optic probes for Raman spectroscopy," Optics Letters 31(12), 1911-1913 (2006).

10. E. C. Le Ru, E. J. Blackie, M. Meyer, and P. G. Etchegoin, "Surface Enhanced Raman Scattering Enhancement Factors: A Comprehensive Study," The Journal of Physical Chemistry C 111(37), 13794-13803 (2007).

11. K. Kneipp, H. Kneipp, R. Manoharan, E. B. Hanlon, I. Itzkan, R. R. Dasari, and M. S. Feld, "Extremely large enhancement factors in surface-enhanced Raman scattering for molecules on colloidal gold clusters," Applied Spectroscopy 52(12), 1493-1497 (1998).

12. P. Mosier-Boss, "Review of SERS Substrates for Chemical Sensing," Nanomaterials 7(6), 142 (2017). 
13. B. Sharma, M. Fernanda Cardinal, S. L. Kleinman, N. G. Greeneltch, R. R. Frontiera, M. G. Blaber, G. C. Schatz, and R. P. Van Duyne, "High-performance SERS substrates: Advances and challenges," MRS Bulletin 38(8), 615624 (2013).

14. L. Yang, P. Li, and J. Liu, "Progress in multifunctional surface-enhanced Raman scattering substrate for detection," RSC Advances 4(91), 49635-49646 (2014).

15. J. C. Hulteen, R. P. Van Duyne, "Nanosphere lithography: A materials general fabrication process for periodic particle array surfaces," Journal of Vacuum Science \& Technology A: Vacuum, Surfaces, and Films 13(3), 15531558 (1995).

16. P. Colson, C. Henrist, and R. Cloots, "Nanosphere Lithography: A Powerful Method for the Controlled Manufacturing of Nanomaterials," Journal of Nanomaterials 2013, 1-19 (2013).

17. J. A. Dieringer, A. D. McFarland, N. C. Shah, D. A. Stuart, A. V. Whitney, C. R. Yonzon, M. A. Young, X. Zhang, and R. P. Van Duyne, "Introductory Lecture: Surface enhanced Raman spectroscopy: new materials, concepts, characterization tools, and applications," Faraday Discussions 132, 9-26 (2006).

18. C. L. Haynes, A. D. McFarland, L. Zhao, R. P. Van Duyne, G. C. Schatz, L. Gunnarsson, J. Prikulis, B. Kasemo, and M. Käll, "Nanoparticle Optics: The Importance of Radiative Dipole Coupling in Two-Dimensional Nanoparticle Arrays ${ }^{\dagger}$, " The Journal of Physical Chemistry B 107(30), 7337-7342 (2003).

19. D. J. White, and P. R. Stoddart, "Nanostructured optical fiber with surface-enhanced Raman scattering functionality," Optics Letters 30(6), 598-600 (2005).

20. J. S. Hartley, S. Juodkazis, and P. R. Stoddart, "Optical fibers for miniaturized surface-enhanced Raman-scattering probes," Applied Optics 52(34), 8388-93 (2013).

21. Y. Liu, Z. Huang, F. Zhou, X. Lei, B. Yao, G. Meng, and Q. Mao, "Highly sensitive fibre surface-enhanced Raman scattering probes fabricated using laser-induced self-assembly in a meniscus," Nanoscale 8(20), 10607-10614 (2016).

22. Z. Yin, Y. Geng, Q. Xie, X. Hong, X. Tan, Y. Chen, L. Wang, W. Wang, and X. Li, "Photoreduced silver nanoparticles grown on femtosecond laser ablated, D-shaped fiber probe for surface-enhanced Raman scattering," Applied Optics 55(20), 5408-5412 (2016).

23. D. L. Stokes, T. Vo-Dinh, "Development of an integrated single-fiber SERS sensor," Sensors and Actuators B: Chemical 69(1-2), 28-36 (2000).

24. M. Pisco, F. Galeotti, G. Quero, G. Grisci, A. Micco, L. V. Mercaldo, P. D. Veneri, A. Cutolo, and A. Cusano, "Nanosphere lithography for optical fiber tip nanoprobes," Light: Science \& Applications 6(5), 1-13 (2017).

25. G. Quero, G. Zito, S. Managò, F. Galeotti, M. Pisco, A. C. De Luca, and A. Cusano, "Nanosphere lithography on fiber: Towards engineered lab-on-fiber SERS optrodes," Sensors 18(3), 680 (2018).

26. X. Zhang, C. R. Yonzon, M. A. Young, D. A. Stuart, and R. P. Van Duyne, "Surface-enhanced Raman spectroscopy biosensors: excitation spectroscopy for optimisation of substrates fabricated by nanosphere lithography," IEEE Proc. - Nanobiotechnology 152(6), 195 (2005).

27. L. F. Santos, R. Wolthuis, S. Koljenović, R. M. Almeida, and G. J. Puppels, "Fiber-optic probes for in vivo Raman spectroscopy in the high-wavenumber region," Analytical Chemistry 77(20), 6747-6752 (2005).

28. A. U. Alam, M. M. R. Howlader, and M. J. Deen, "The effects of oxygen plasma and humidity on surface roughness, water contact angle and hardness of silicon, silicon dioxide and glass," Journal of Micromechanics and Microengineering 24(3), 035010 (2014).

29. J. F. Rusling, "Minimizing errors in numerical analysis of chemical data," Journal of Chemical Education 65(10), 863 (1988). 